\title{
Comparison of three methods for the estimation of cross-shock electric potential using Cluster data
}

\author{
A. P. Dimmock ${ }^{1}$, M. A. Balikhin ${ }^{1}$, and Y. Hobara ${ }^{2}$ \\ ${ }^{1}$ Automatic Control \& Systems Engineering, University of Sheffield, Sheffield, UK \\ ${ }^{2}$ Department of Communication Engineering and Informatics, The University of Electro-Communications, Tokyo, Japan
}

Received: 28 February 2011 - Revised: 15 April 2011 - Accepted: 18 April 2011 - Published: 13 May 2011

\begin{abstract}
Cluster four point measurements provide a comprehensive dataset for the separation of temporal and spatial variations, which is crucial for the calculation of the cross shock electrostatic potential using electric field measurements. While Cluster is probably the most suited among present and past spacecraft missions to provide such a separation at the terrestrial bow shock, it is far from ideal for a study of the cross shock potential, since only 2 components of the electric field are measured in the spacecraft spin plane. The present paper is devoted to the comparison of 3 different techniques that can be used to estimate the potential with this limitation. The first technique is the estimate taking only into account the projection of the measured components onto the shock normal. The second uses the ideal MHD condition $\boldsymbol{E} \cdot \boldsymbol{B}=0$ to estimate the third electric field component. The last method is based on the structure of the electric field in the Normal Incidence Frame (NIF) for which only the potential component along the shock normal and the motional electric field exist. All 3 approaches are used to estimate the potential for a single crossing of the terrestrial bow shock that took place on the 31 March 2001. Surprisingly all three methods lead to the same order of magnitude for the cross shock potential. It is argued that the third method must lead to more reliable results. The effect of the shock normal inaccuracy is investigated for this particular shock crossing. The resulting electrostatic potential appears too high in comparison with the theoretical results for low Mach number shocks. This shows the variability of the potential, interpreted in the frame of the non-stationary shock model.
\end{abstract}

Keywords. Space plasma physics (Electrostatic structures; Shock waves)

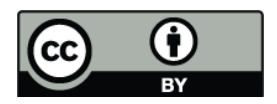

Correspondence to: A. P. Dimmock (a.dimmock@sheffield.ac.uk)

\section{Introduction}

The main process that takes place at the front of a collisionless shock is the redistribution of the upstream ion flow kinetic energy into the heating of the bulk plasma, and the acceleration of a fraction of plasma particles to very high energies. Since the very early stage of shock physics it was argued that the interactions between plasma particles and fields in the shock front replaces the role of collisions in ordinary hydrodynamic shocks and leads to the energy redistribution at the front (Sagdeev, 1966; Sagdeev and Galeev, 1969). In many cases such interactions were explained in the frame of anomalous processes related to various instabilities in the shock front. A comprehensive review of various instabilities in the shock front can be found in Papadopoulos (1985). Results of the extensive experimental studies of the terrestrial bow shock by INTERSHOCK and AMPTE (Scudder et al., 1986; Krasnoselskikh et al., 1991) and numerical simulations (Leroy et al., 1982; Scholer et al., 2003) have shown that the effect of coherent macroscopic fields in the front of a strong, supercritical, quasi-perpendicular shock is enough to account for observed plasma thermalisation and acceleration. Recent observations by Venus Express (VEX) have shown that the effect of this force is enough to explain thermalisation in weak quasi-perpendicular shocks as well (Balikhin et al., 2008). This does not mean that early models based on the quest of micro instabilities that take the place of collisions to allow shocks to form (Sagdeev, 1966) have lost their importance. On the contrary, such instabilities are very important for the energy redistribution at quasi-parallel shocks. Moreover, the main motivation in shock studies is that they are the most effective accelerators. According to present views, cosmic rays consist of particles accelerated to huge energies by collision-less shocks formed in the vicinity of various astrophysical objects. These accelerated particles and waves generated then determine the structure of these astrophysical shocks. In addition, numerical simulations for

Published by Copernicus Publications on behalf of the European Geosciences Union. 
ultra-relativistic shocks associated with gamma ray burst afterglow indicate such shocks are formed by filamentational instability as in classical anomalous process based shock models.

However, as mentioned above for the quasi-perpendicular planetary and interplanetary shocks that are observed in the solar system, macro electric and magnetic fields in the shock front can explain the energy redistribution without invoking models based on instabilities (Wu, 1984; Leroy and Mangeney, 1984; Scudder et al., 1986; Balikhin and Gedalin, 1994). Therefore comprehensive measurements of electrostatic potential and the magnetic field structure of the shock front are required to understand the evolution of the plasma parameters across these shocks. The number of studies devoted to the magnetic field structure of the terrestrial bow shock significantly outnumber the studies of the electric field. One of the possible reasons is due to the complexity of the electric field measurements across the region with nonuniform plasma temperature/density. Only a few papers devoted to the electric field and electrostatic potential in the shock have been published (Heppner et al., 1978; Wygant et al., 1987; Formisano, 1982; Scudder et al., 1986; Balikhin et al., 2002; Scholer et al., 2003) in comparison to hundreds dedicated to the magnetic field structure of the various types of shocks. The estimate of the cross shock potential is also susceptible to the inaccuracy of the calculated relative shock/spacecraft velocity, because it requires the spacial integration of the electric field over the spatial coordinates. Therefore, the ability to distinguish between temporal and spatial variations is crucial for the reliable identification of the shock front potential. Four closely spaced satellites such as Cluster appear ideal for the analysis of the shock potential. However, the electric field instrument onboard each of the Cluster satellites does not measure all three components of the electric field, providing only the $\mathrm{X}$ - and Y-components in the satellite spin plane. In order to exploit the spatiotemporal potential of the Cluster mission, additional assumptions are required to estimate the potential in such cases. A straight-forward approach which involves no computation prior to the calculation of the potential assumes that if the angle between the spin plane and the shock normal is small, then the potential can be estimated using only the two available electric field components. If the spin plane is not almost perpendicular to the shock normal such an estimate should give a correct order of magnitude for the cross shock potential. The second method that has been used is based on the assumption $\boldsymbol{E} \cdot \boldsymbol{B}=0$ (ideal MHD). This condition allows the identification of the third component of the electric field and subsequently the cross shock potential. This methodology has been used in a number of studies (Bale and Mozer, 2007; Bale et al., 2008). The final method to be considered in the present study uses the structure of electric field in the Normal Incidence Frame (NIF) in which the upstream velocity lies along the shock normal. Only two components of electric field exist in this frame: the potential along the shock normal, and $\boldsymbol{V} \times \boldsymbol{B}$. As velocity is directed along the normal, only the component of the magnetic field that is perpendicular to the shock normal $B_{\text {perp }}$ contributes to the term $\boldsymbol{V} \times \boldsymbol{B}$. Therefore $\boldsymbol{E} \cdot B_{\text {perp }}$ must be equal to zero, giving the possibility to determine the missing third component of the electric field and therefore identify the cross-shock potential. The present paper is devoted to the comparative study of these 3 methods applied to a particular shock observed by the four Cluster spacecraft on 31 March 2001.

\section{Data and instrumentation}

The data used in this study were collected by the Cluster spacecraft during a day of 11 bow shock crossings on 31 March 2001. The electric field measurements were made by the Electric Fields and Waves experiment (EFW) (Gustafsson et al., 1997), which is part of the wave consortium controlled by the Digital Wave Processor (DWP) (Woolliscroft et al., 1997). The EFW instrument consists of 4 spherical probes deployed on $44 \mathrm{~m}$ wire booms $(88 \mathrm{~m}$ sensor separation), the potential difference between the probes is used to measure the electric field components in the spin plane of the spacecraft (ISR2). In the ISR2 frame, the spacecraft spin axis is represented by the $\mathrm{X}$-axis. When the ISR2 frame is inverted about the spin axis it varies by $<6^{\circ}$ of the geocentric solar ecliptic (GSE) frame. A significant limitation of this instrument is the absence of a third field vector, as a result only 2 components are recorded in the ISR2 frame. The Fluxgate Magnetometer (FGM) instrument (Balogh et al., 1997) provides magnetic field measurements which are used to identify the shock crossing region, and correlate with the EFW datasets. The time resolution of the EFW and FGM datasets are $25 \mathrm{~Hz}$ and $22 \mathrm{~Hz}$ respectively. Ion density $\left(N_{i}\right)$ used to calculate Alfvén Mach number $\left(M_{\mathrm{a}}\right)$, was estimated using the electron plasma frequency $\left(\omega_{p e}\right)$ measured by the WHISPER instrument (Décréau et al., 1997). The solar wind upstream bulk flow velocity $\left(V_{\text {up }}\right)$ was obtained from the Cluster Ion Spectrometer (CIS) instrument (Rème et al., 1997).

\section{Shock crossing: 31 March 2001, 18:28 UT}

The present paper is devoted to a particular shock that occurred on 31 March 2001 at 18:28 UT. On this day solar wind conditions were to some extent irregular due to the passage of a CME. The magnetic field and solar wind velocity upstream of the shock measured by the Cluster 1 spacecraft were $27 \mathrm{nT}$ and $590 \mathrm{~km} \mathrm{~s}^{-1}$, respectively. The model normal (Farris et al., 1991) in the GSE frame is [0.92, -0.09, 0.37], and the shock velocity was determined to be $29 \mathrm{~km} \mathrm{~s}^{-1}$. Remaining parameters are $\theta_{\mathrm{BN}}=88^{\circ}$ and plasma density $N_{i}=$ $17.4 \mathrm{~cm}^{-3}$. The Alfvén Mach number is rather moderate at 3.7 which is consistent with the lack of any significant overshoot. 

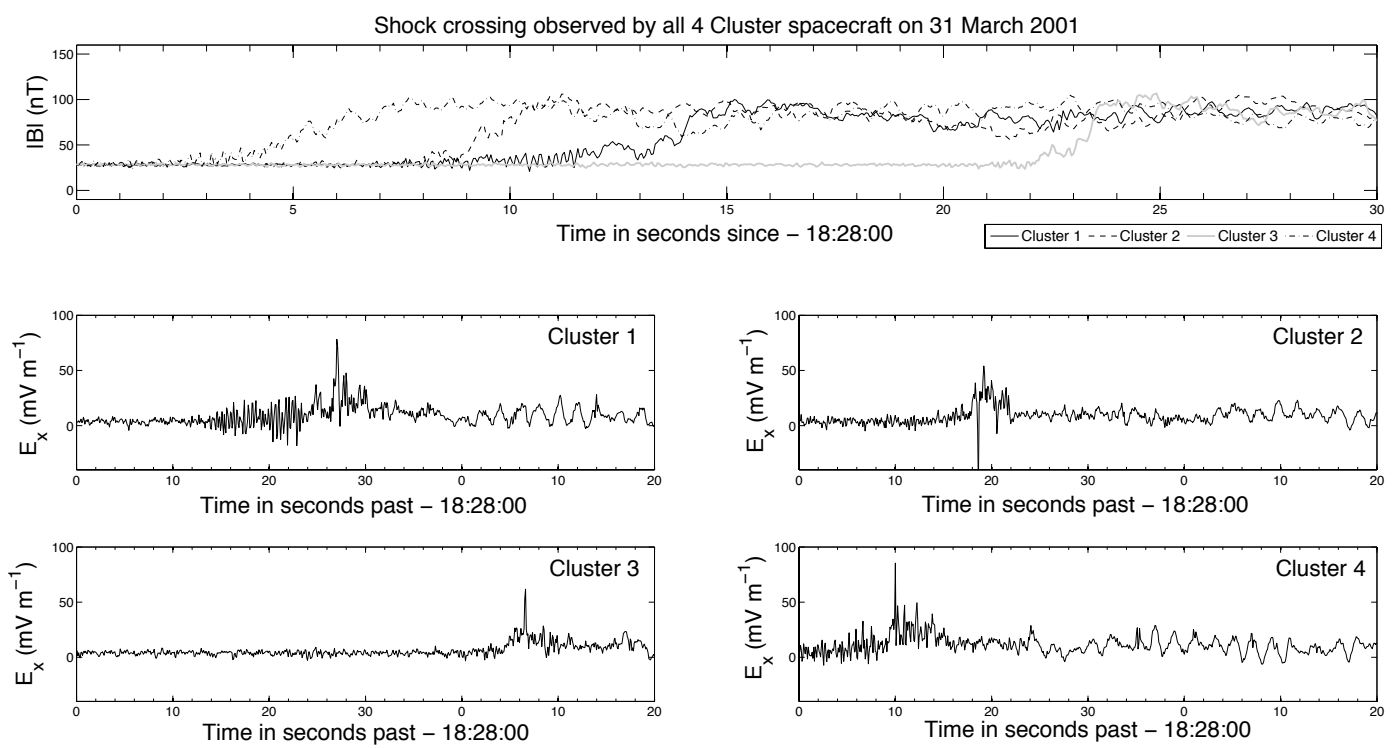

Fig. 1. Measurements made by the four Cluster spacecraft as they observed one of eleven bow shock crossing on 31 March 2001 at 18:28 UT. The top panel illustrates the magnitude of the magnetic field profile measured by the four FGM instruments onboard each spacecraft. The four lower panels show the electric field measurements recorded by the relative EFW instruments over the same time period.
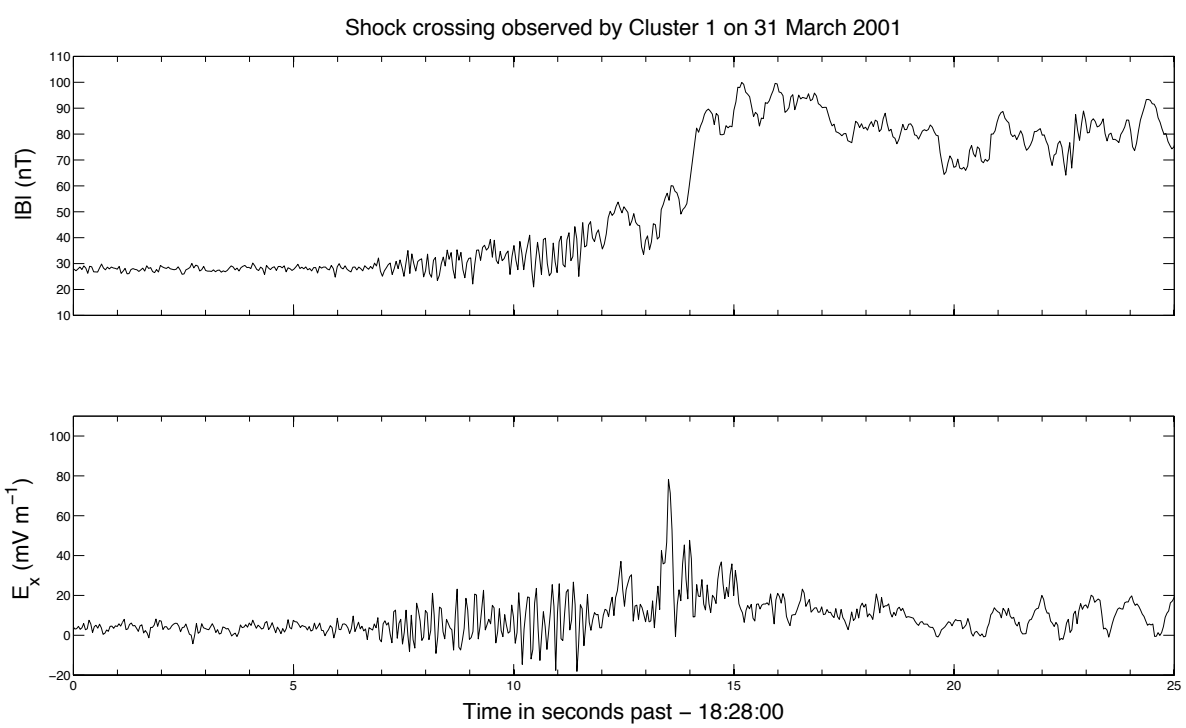

Fig. 2. A shock crossing observed by the Cluster 1 spacecraft at 18:28 on 31 March 2001. The upper panel shows the magnitude of the magnetic field profile measured the Cluster 1 FGM instrument across the shock. The lower panel shows the x-component of the electric field recorded by the EFW instrument over the same time interval (in the spacecraft spin frame).

The sequence in which the Cluster spacecraft encountered the bow shock was $\mathrm{C} 4, \mathrm{C} 2, \mathrm{C} 1$ and finally $\mathrm{C} 3$. This is illustrated by the top panel of Fig. 1. Additionally Fig. 2 shows the measurements during the shock crossing recorded by the FGM and EFW instruments onboard the Cluster 1 spacecraft. The top panel of Fig. 2 displays the magnitude of the magnetic field several seconds before and after the shock. The magnetic profile displays an abundance of low frequency plasma waves prior to the shock crossing which commence at approximately 18:28:07 UT. The lower panel illustrates the $\mathrm{X}$-component of the EFW measurements in the spacecraft spin frame. The electric field appears constant upstream of the shock at around $5 \mathrm{mV} \mathrm{m}^{-1}$ which reflects the $\boldsymbol{V} \times \boldsymbol{B}$ term. It is worth noting that fluctuations within the electric field appear to accurately correspond to observations in the magnetic field profile. There is also a notable spike like structure at 18:28:13.500 UT during the crossing. Such small scale structures were often observed within the quasi-perpendicular part 

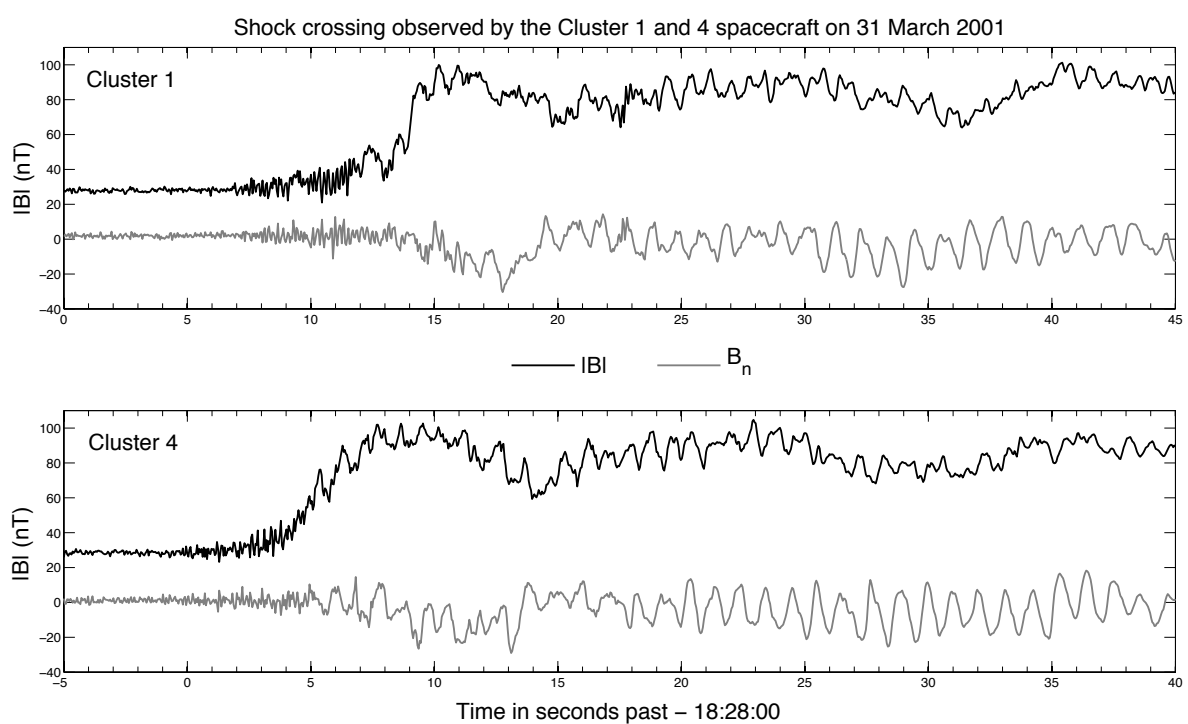

Fig. 3. The magnetic field profile of a shock crossing observed by the Cluster 1 and 4 satellites on 31 March 2001. The time interval shows the crossing several seconds upstream and around $30 \mathrm{~s}$ downstream of the crossing. The black line shows the magnitude of the magnetic field whereas the grey line is the magnetic field projected along the normal. The upper and lower panels represent the Cluster 1 and Cluster 4 spacecraft, respectively.

of the terrestrial bow shock and have been statistically studied by Walker et al. (2004). The lower panels of Fig. 1 demonstrate that a small scale structure within the shock front, has been observed in the electric field by all four Cluster spacecraft.

\section{Shock normal}

The shock normal $\hat{\boldsymbol{n}}$ is one of the key parameters in the estimate of the cross-shock potential $\phi$. This is not only because it is the electric field component parallel to $\hat{\boldsymbol{n}}\left(E_{n}\right)$ that contributes to $\phi$, but also due to the effect of the normal shockspacecraft velocity on the spatial integration of the electric field. In the present paper the shock normal $n$ has been identified using the Farris et al. (1991) model shock surface. The multi spacecraft timing analysis (Schwartz, 1998) produces a normal that has a very small angle $\left(<5^{\circ}\right)$ with $\hat{\boldsymbol{n}}$. Figure 3 displays $|B|$ and the projection of the magnetic field along $\hat{\boldsymbol{n}}\left(B_{n}\right)$, for the Cluster 1 (top) and Cluster 4 (bottom) spacecraft. It can be seen that the average values of $B_{n}$ do not possess any significant change within the ramp where $|B|$ experiences a 60-70 nT change. The change of the average value from the far upstream to the deep downstream is also insignificant, supporting the estimate of the normal $\hat{\boldsymbol{n}}$. Spacecraft 2 and 3 show similar results. However, for all four spacecraft the decreasing portions of overshot coincide with deviation in $B_{n}$ as also can be seen in Fig. 3. This can be explained by a presence of an additional ripple-like local structure.
The velocity along the shock normal was determined based on a selection of the 6 possible geometric parings of the 4 Cluster satellites as they encountered the bow shock. Only 3 pairs of crossings have been used, since the other 3 separation vectors were close to being perpendicular to $\hat{\boldsymbol{n}}$. The following 3 spacecraft pairings were used, $\mathrm{C} 1 \rightarrow \mathrm{C} 4$, $\mathrm{C} 2 \rightarrow \mathrm{C} 3$ and $\mathrm{C} 3 \rightarrow \mathrm{C} 4$. The total variation between the 3 identified velocities was less that $15 \%$. The mean of the 3 velocity pairings $29.4 \mathrm{~km} \mathrm{~s}^{-1}$ has been used as the shock velocity $V_{\mathrm{s}}$.

\section{Methodology for the estimation of cross-shock potential}

As the electric field is frame dependent, the Lorentz transformation should be used to estimate the cross shock potential in the NIF frame using the electric field data measured in the spacecraft frame. The electric field components resulting from $V_{\mathrm{s}}$ and $\boldsymbol{V}_{\text {nif }}\left(\mathrm{NIF}\right.$ frame velocity $\left.\boldsymbol{V}_{\text {nif }}=\hat{\boldsymbol{n}} \times\left(\boldsymbol{V}_{u} \times \hat{\boldsymbol{n}}\right)\right)$ could reach quite significant values of a few $\mathrm{mV} \mathrm{m}^{-1}$ which may contribute errors leading to the miscalculation of $\phi$.

Electric field measurements made by the EFW instrument onboard all 4 Cluster spacecraft consist of only 2 components directed along the $\mathrm{X}$ and $\mathrm{Y}$ directions in the spacecraft spin frame. As a result only an estimate of $\phi$ can be calculated. The present paper is devoted to three separate techniques for estimating cross shock potential.

The estimate of the cross shock potential $\phi_{\text {est }}$ can be obtained by taking into account only the two components measured by the EFW instrument. The implicit assumption in 


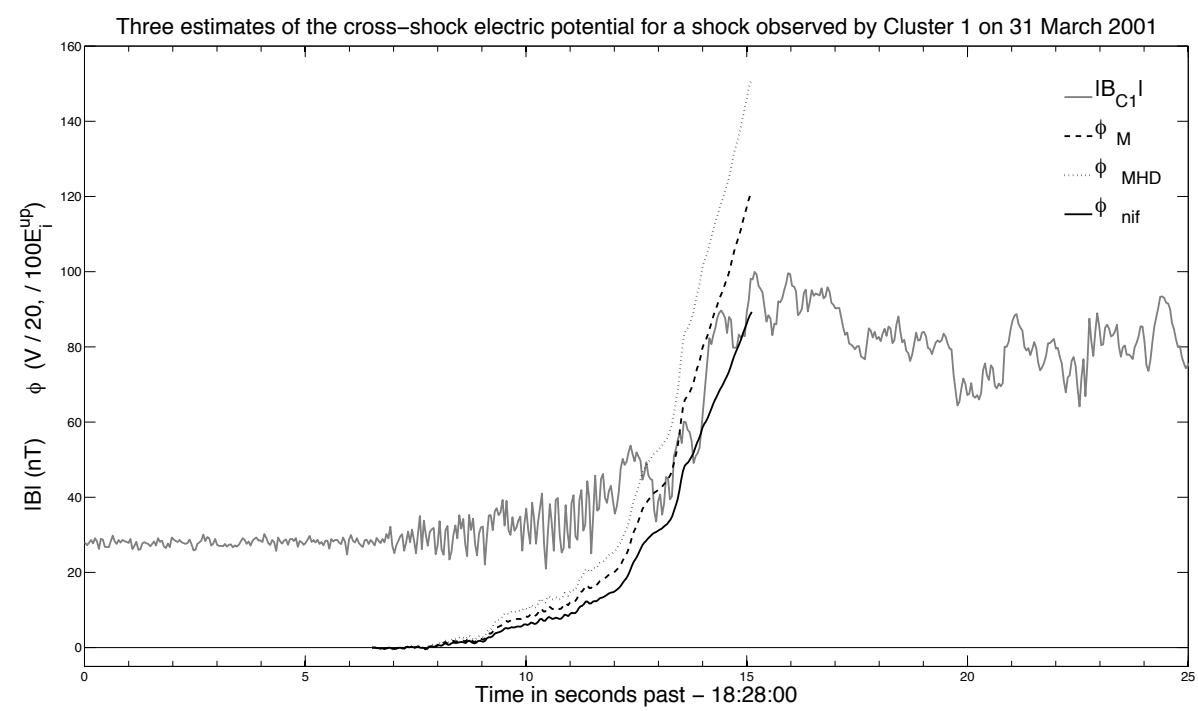

Fig. 4. A shock crossing made by the Cluster 1 spacecraft around 18:28 on 31 March 2001. The grey line shows the magnetic field magnitude measured by the Cluster 1 FGM instrument during the bow shock crossing. The dotted black line is the estimate based on the assumption that the electric field along $\boldsymbol{B}$ is zero. The black dashed line shown the estimate based on only the 2 measured electric field components. The black solid line represents the potential estimate by evaluating the missing electric field component based on the NIF condition $\left(E_{\mathrm{NIF}} \cdot B_{\perp}=0\right)$.

this procedure is that the direction of the shock normal is not almost perpendicular to the axis of the spacecraft spin plane. Such an estimate will provide a correct spatial scale of the cross shock potential and a reasonable estimate of its magnitude. However, this method cannot be expected to produce a precise magnitude of the potential $|\phi|$.

To obtain more reliable and accurate values of the cross shock potential from Cluster data, the properties of the electric field in the NIF frame $E_{\mathrm{NIF}}$ of reference can be used. In the NIF the motional component $\boldsymbol{V}_{u} \times \boldsymbol{B}$ is perpendicular to the electrostatic component which is the gradient of $\phi$ along the normal. The upstream magnetic field can be decomposed into the component parallel to $\hat{\boldsymbol{n}}\left(B_{n}\right)$, and a perpendicular component $B_{\perp}$. The condition $E_{\mathrm{NIF}} \cdot B_{\perp}=0$ allows the determination of the third unmeasured component of the electric field.

Often when only two components of the electric field are available the third component is reconstructed by assuming that the component of $\boldsymbol{E}$ along $\boldsymbol{B}$ is zero (Bale and Mozer, 2007) $\boldsymbol{E} \cdot \boldsymbol{B}=0$ (ideal MHD). It is worth noting that whilst this approximation might provide an accurate estimate for some other structures and regions, it is unacceptable for the terrestrial bow shock. This can be illustrated by electron dynamics. The de-Hoffman-Teller frame (HTF) of reference is defined by the condition that the upstream velocity is parallel to the upstream magnetic field. Therefore the motional component of electric field vanishes, leading to charged particle energy conservation in the HTF. As discussed by Goodrich and Scudder (1984) the electrostatic potential in the HTF is directly related to the electron energization. Setting the parallel electric field to zero will distort the value of the electro- static potential in HTF for quasi-perpendicular shocks. As a result the "bump on flattop" electron distributions (Feldman et al., 1983) would not be observed. In spite of all this criticism of the $\boldsymbol{E} \cdot \boldsymbol{B}=0$ assumption, it will be used in the present paper for comparison with the results obtained by the first two methods.

It is worth noting that upstream of the shock front the only component that contributes to the DC electric field is the motional $\boldsymbol{V} \times \boldsymbol{B}$ field. This value will be constant across the shock. Therefore, the upstream value of $\boldsymbol{V} \times \boldsymbol{B}$ can be used to account for the motional electric field across the whole shock front.

Finally to calculate the electrostatic potential, $E_{\mathrm{NIF}}$ is spatially integrated through the shock front, including both the foot and shock ramp regions. The integration is discontinued at the end of the shock ramp just prior to downstream.

\section{Results}

The change of the electrostatic potential within the shock crossing measured by the Cluster 1 spacecraft, is displayed in Fig. 4, together with the magnitude of the magnetic field (grey solid line). The zero level reference line of $\phi=0$ is also shown in this figure. Three methods of potential estimates lead to the differences in the $\phi$. The lowest value of the potential is a result of the method based on the NIF condition $E_{\mathrm{NIF}} \cdot B_{\perp}=0$ (solid line). The highest is based on the ideal MHD assumption previously used by Bale et al. (2008) (dotted). The estimate based on 2 components only results in the intermediate estimate (dashed). The legend in this figure describes the scaling of the potential with respect to both 
Table 1. Cross shock potential estimates for each electric field dataset. Provided are calculations for the potential in Volts and also the potential normalised with respect to the upstream ion kinetic energy $\left(E_{i}^{\mathrm{up}}\right)$.

\begin{tabular}{lcccccccc}
\hline Mehod & $C_{1} \phi(\mathrm{V})$ & $C_{1} \phi\left(/ E_{i}^{\mathrm{up}}\right)$ & $C_{2} \phi(\mathrm{V})$ & $C_{2} \phi\left(/ E_{i}^{\mathrm{up}}\right)$ & $C_{3} \phi(\mathrm{V})$ & $C_{3} \phi\left(/ E_{i}^{\mathrm{up}}\right)$ & $C_{4} \phi(\mathrm{V})$ & $C_{4} \phi\left(/ E_{i}^{\mathrm{up}}\right)$ \\
\hline NIF structure & 1785 & 0.9042 & 1330 & 0.6737 & 563 & 0.2852 & 1991 & 1.0086 \\
2 Measured Components & 2426 & 1.2290 & 1796 & 0.9098 & 762 & 0.3860 & 2703 & 1.3693 \\
$E . B=0$ (ideal MHD) & 3026 & 1.5329 & 2147 & 1.0876 & 858 & 0.4346 & 3326 & 1.6849 \\
\hline
\end{tabular}

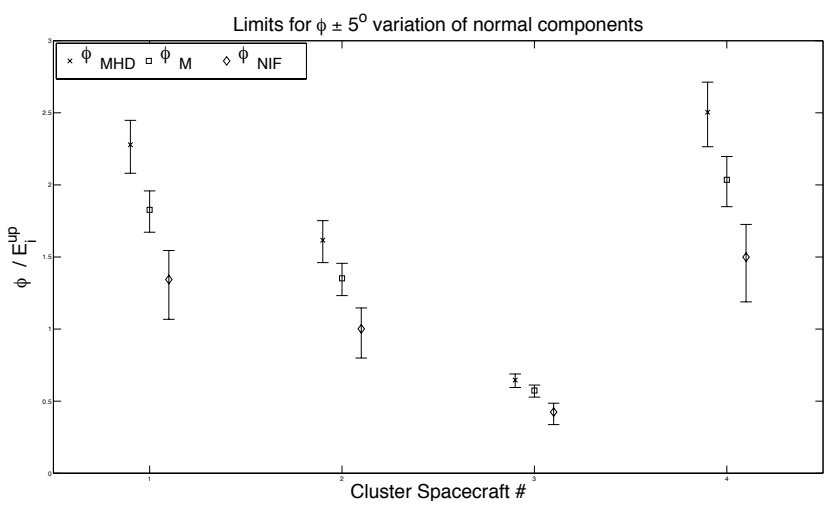

Fig. 5. Electric cross-shock potential estimates of all four Cluster spacecraft. The circle markers represent the potential estimates prior to any variation whereas, the error bars represent the upper and lower limits of the maximum and minimum potential evaluations as a result of a variation about the model normal of $\pm 5^{\circ}$.

Volts and the upstream ion energy $E_{i}^{\mathrm{up}}=\frac{m_{i} V_{\mathrm{up}}^{2}}{2}$. Table 1 summarises the values of the overall cross shock potential change obtained by these 3 techniques for all four Cluster spacecraft. It can be seen that the relative values of the potential estimate by all 3 methods are similar to these obtained by the Cluster 1 spacecraft. The lowest and highest values are always resulting from $E_{\mathrm{NIF}} \cdot B_{\perp}=0$ and $\boldsymbol{E} \cdot \boldsymbol{B}=0$, respectively. The 2 component based estimate values are always intermediate with respect to the other methods. Even the lowest of the potential estimates obtained using NIF condition appear too high in the case of the Cluster 1 and 4 crossings. The possible physical reasons for such high values of the potential will be explained later in the Discussion section. To ensure that these values are not the result of an error during the identification of the shock normal and consequent shock velocity, the effect of the normal variation has been investigated for the NIF condition based method. The direction of the normal $\hat{\boldsymbol{n}}$ has been subjected to a variation of a $10^{\circ}$ cone around its identified model value. Obviously any variation in the direction of the normal leads to variation in the shock velocity, NIF frame, $E_{\mathrm{NIF}}$ etc. The extreme minimal and maximum values resulting from such a variation within the cone are shown as the upper and lower boundaries for the error bars in Fig. 5. It can be seen that even minimal values of the potential for
Cluster 4 resulting from such variation is quite high at about $80 \%$ of the upstream ion kinetic energy.

\section{Discussion}

The change of the cross shock potential for Cluster 1 displayed in Fig. 4 is representative of the four spacecraft crossings of this shock. The increase in the potential starts upstream of the ramp in the region of low frequency turbulence. Since ISEE and AMPTE projects, it is known that this region almost coincides with the foot of a quasi-perpendicular shock (e.g. Krasnoselskikh et al., 1991). Initially it was thought that these waves are the result of plasma instabilities caused by the counter streaming plasma flow and the beam of reflected ions. However, data from closely spaced spacecraft (inside the coherency length of the turbulence) enabled the dismissal of these models and indicated that these waves are the result of the nonlinear evolution of the shock front itself (Krasnoselskikh, 1985; Balikhin et al., 1997, 1999; Walker et al., 2008). The increase of the potential in the foot is about a quarter of the overall change. The rest of the increase corresponds to the region of the magnetic ramp. A small scale structure is evident in the electric field at around 18:28:13.5 in Fig. 1 which contributes around $15 \%$ of the electrostatic potential. According to the estimation based on the NIF condition, the contribution of this small scale structure is around $300 \mathrm{~V}$. Such a considerable increase of the potential over a small spatial scale should lead to non-adiabatic dynamics of electrons and a corresponding increase of temperature (Gedalin et al., 1995; Balikhin and Gedalin, 1994; Balikhin et al., 1998). The increase of the cross shock potential should also lead to the decrease of the ion thermal energy downstream of the shock e.g. Ofman et al. (2009).

The peculiar feature of the potential estimates for all four shocks is the unexpectedly high values of $\phi$ in comparison to theoretical studies such as Gedalin (1997). In a study by Bale et al. (2008) of the bow shock crossings that take place on 31 March 2001, their methodology (ideal MHD) also produced high potential estimates very similar to the results obtained with the ideal MHD condition in the present paper. The overall high resulting potential for the shock studied in the present paper can be the result of two factors. The first is the high concentration of alpha particles on that day (about $9 \%$ of the 
protons). Therefore the value of the upstream average ion kinetic energy based on the proton mass should lead to significant underestimation. The second reason is the unusual CME observed on this day. There are a total of 9 crossings of the terrestrial bow shock in a short period of about 2 and a half hours. This indicates non-stationarity of the solar wind conditions. Such non-stationarity can lead to shock reformation induced by the change in the abnormal solar wind conditions, and results in unusual values of the potential for this particular crossing.

The main conclusion that should be drawn from this study is that all three methods lead to the same order of magnitude of the cross shock potential, and exactly the same spatial scales of the potential change. However as these methods still lead to a significant difference in the potential estimates, the NIF derived method should be used for a more accurate estimation. As the $\boldsymbol{E} \cdot \boldsymbol{B}=0$ technique is based on an assumption that is not valid at the shock front. The simplistic methodology of the potential estimate when only two measured components are taken into account (without any other additional assumptions) are able to provide the same reliability of $\phi$ spatial scales as the more sophisticated technique that uses the NIF condition $E_{\mathrm{NIF}} \cdot B_{\perp}=0$. The spatial scales of the shock are one of the most important parameters, as they are related to the physical processes that balance nonlinearity and lead to the shock structure formation (Kennel et al., 1985; Sagdeev, 1979; Papadopoulos, 1981). In addition, the spatial scale determines the mechanism of interaction between the incoming solar wind particles, and the macro electric and magnetic fields within the shock. While there are many studies of the magnetic field scales within the shock front (e.g. Balikhin et al., 1995; Hobara et al., 2010; Newbury and Russell, 1996), only a few studies are devoted to the scales of the electric field e.g. Walker et al. (2004). The results of the present study facilitate the ability to estimate $\phi$ spatial scales in the case of limited electric field datasets such as Cluster, and can allow an easier comprehensive statistical study of these scales based on a large number of shocks observed by Cluster.

Acknowledgements. This work was supported by STFC and EPSRC grants. The authors wish to acknowledge the Custer FGM, EFW, CIS, WHISPER and DWP teams for providing the datasets for this study.

Guest Editor M. Gedalin thanks L. Ofman and another anonymous referee for their help in evaluating this paper.

\section{References}

Bale, S. D. and Mozer, F. S.: Measurement of Large Parallel and Perpendicular Electric Fields on Electron Spatial Scales in the Terrestrial Bow Shock, Phys. Rev. Lett., 98, 205001, doi:10.1103/PhysRevLett.98.205001, 2007.

Bale, S. D., Mozer, F. S., and Krasnoselskikh, V. V.: Direct measurement of the cross-shock electric potential at low plasma $\beta$, quasi-perpendicular bow shocks, ArXiv e-prints, 2008.
Balikhin, M. and Gedalin, M.: Kinematic mechanism of electron heating in shocks: Theory vs observations, Geophys. Res. Lett., 21, 841-844, doi:10.1029/94GL00371, 1994.

Balikhin, M., Krasnosselskikh, V., and Gedalin, M.: The scales in quasiperpendicular shocks, Adv. Space Res., 15, 247-260, doi:10.1016/0273-1177(94)00105-A, 1995.

Balikhin, M. A., Walker, S. N., de Wit, T. D., Alleyne, H. S. C. K., Woolliscroft, L. J. C., Mier-Jedrzejowicz, W. A. C., and Baumjohann, W.: Non-stationarity and low frequency turbulence at a quasiperpendicular shock front, Adv. Space Res., 20, 729-734, doi:10.1016/S0273-1177(97)00463-8, 1997.

Balikhin, M., Krasnoselskikh, V. V., Woolliscroft, L. J. C., and Gedalin, M.: A study of the dispersion of the electron distribution in the presence of $\mathrm{E}$ and $\mathrm{B}$ gradients: Application to electron heating at quasi-perpendicular shocks, J. Geophys. Res., 103, 2029-2040, doi:10.1029/97JA02463, 1998.

Balikhin, M. A., Alleyne, H., Treumann, R. A., Nozdrachev, M. N., Walker, S. N., and Baumjohann, W.: The role of nonlinear interaction in the formation of LF whistler turbulence upstream of a quasi-perpendicular shock, J. Geophys. Res. (Space Physics), 104, 12525-12536, doi:10.1029/1998JA900102, 1999.

Balikhin, M. A., Nozdrachev, M., Dunlop, M., Krasnoselskikh, V., Walker, S. N., Alleyne, H. S. C. K., Formisano, V., Andre, M., Balogh, A., Eriksson, A., and Yearby, K.: Observation of the terrestrial bow shock in quasi-electrostatic subshock regime, J. Geophys. Res. (Space Physics), 107, 1155, doi:10.1029/2001JA000327, 2002.

Balikhin, M. A., Zhang, T. L., Gedalin, M., Ganushkina, N. Y., and Pope, S. A.: Venus Express observes a new type of shock with pure kinematic relaxation, Geophys. Res. Lett., 35, L01103, doi:10.1029/2007GL032495, 2008.

Balogh, A., Dunlop, M. W., Cowley, S. W. H., Southwood, D. J., Thomlinson, J. G., Glassmeier, K. H., Musmann, G., Lühr, H., Buchert, S., Acuña, M. H., Fairfield, D. H., Slavin, J. A., Riedler, W., Schwingenschuh, K., and Kivelson, M. G.: The Cluster magnetic field investigation, Space Sci. Rev., 79, 65-91, 1997.

Décréau, P. M. E., Fergeau, P., Kranoselskikh, V., Lévêque, M., Martin, P., Randriamboarison, O., Sené, F. X., Trotignon, J. G., Canu, P., and Mögensen, P. B.: WHISPER, a resonance sounder and wave analyser: performances and perspectives for the Cluster mission, Space Sci. Rev., 79, 157-193, 1997.

Farris, M. H., Petrinec, S. M., and Russell, C. T.: The thickness of the magnetosheath - Constraints on the polytropic index, Geophys. Res. Lett., 18, 1821-1824, doi:10.1029/91GL02090, 1991.

Feldman, W. C., Anderson, R. C., Bame, S. J., Gary, S. P., Gosling, J. T., McComas, D. J., Thomsen, M. F., Paschmann, G., and Hoppe, M. M.: Electron velocity distributions near the earth's bow shock, J. Geophys. Res., 88, 96-110, doi:10.1029/JA088iA01p00096, 1983.

Formisano, V.: Measurement of the potential drop across the earth's collisionless bow shock, Geophys. Res. Lett., 9, 1033-1036, doi:10.1029/GL009i009p01033, 1982.

Gedalin, M.: Ion heating in oblique low-Mach number shocks, Geophys. Res. Lett., 24, 2511-2514, doi:10.1029/97GL02524, 1997.

Gedalin, M., Gedalin, K., Balikhin, M., and Krasnosselskikh, V.: Demagnetization of electrons in the electromagnetic field structure, typical for quasi-perpendicular collisionless shock front, J. Geophys. Res., 100, 9481-9488, doi:10.1029/94JA03369, 1995.

Goodrich, C. C. and Scudder, J. D.: The adiabatic energy change 
of plasma electrons and the frame dependence of the cross-shock potential at collisionless magnetosonic shock waves, J. Geophys. Res., 89, 6654-6662, doi:10.1029/JA089iA08p06654, 1984.

Gustafsson, G., Bostrom, R., Holback, B., Holmgren, G., Lundgren, A., Stasiewicz, K., Ahlen, L., Mozer, F. S., Pankow, D., Harvey, P., Berg, P., Ulrich, R., Pedersen, A., Schmidt, R., Butler, A., Fransen, A. W. C., Klinge, D., Thomsen, M., Falthammar, C., Lindqvist, P., Christenson, S., Holtet, J., Lybekk, B., Sten, T. A., Tanskanen, P., Lappalainen, K., and Wygant, J.: The Electric Field and Wave Experiment for the Cluster Mission, Space Sci. Rev., 79, 137-156, doi:10.1023/A:1004975108657, 1997.

Heppner, J. P., Maynard, N. C., and Aggson, T. L.: Early results from ISEE-1 electric field measurements, Space Sci. Rev., 22, 777-789, 1978.

Hobara, Y., Balikhin, M., Krasnoselskikh, V., Gedalin, M., and Yamagishi, H.: Statistical study of the quasi-perpendicular shock ramp widths, J. Geophys. Res. (Space Physics), 115, 11106, doi:10.1029/2010JA015659, 2010.

Kennel, C. F., Edmiston, J. P., and Hada, T.: A quarter century of collisionless shock research, Washington D.C. American Geophysical Union Geophysical Monograph Series, 34, 1-36, 1985.

Krasnoselskikh, V.: Nonlinear motions of a plasma across a magnetic field, Sov. Phys. Jetp, 62, 282-293, 1985.

Krasnoselskikh, V. V., Vinogradova, T., Balikhin, M. A., Alleyne, H. S. C., Pardaens, A. K., Woolliscroft, L. J. C., Klimov, S. I., Petrukovich, A., Mier-Jedrzejowicz, W. A. C., and Southwood, D. J.: On the nature of low frequency turbulence in the foot of strong quasi-perpendicular shocks, Adv. Space Res., 11, 15-18, doi:10.1016/0273-1177(91)90002-2, 1991.

Leroy, M. M. and Mangeney, A.: A theory of energization of solar wind electrons by the earth's bow shock, Ann. Geophys., 2, 449456, 1984

Leroy, M. M., Winske, D., Goodrich, C. C., Wu, C. S., and Papadopoulos, K.: The structure of perpendicular bow shocks, J. Geophys. Res., 87, 5081-5094, doi:10.1029/JA087iA07p05081, 1982.

Newbury, J. A. and Russell, C. T.: Observations of a very thin collisionless shock, Geophys. Res. Lett., 23, 781-784, 1996.

Ofman, L., Balikhin, M., Russell, C. T., and Gedalin, M.: Collisionless relaxation of ion distributions downstream of laminar quasi-perpendicular shocks, J. Geophys. Res., 114, A09106, doi:10.1029/2009JA014365, 2009.

Papadopoulos, K.: Comments on high Mach number magnetosonic shocks, Tech. rep., European Space Agency, 1981.

Papadopoulos, K.: Microinstabilities and anomalous transport, Washington D.C. American Geophysical Union Geophysical Monograph Series, 34, 59-90, 1985.
Rème, H., Bosqued, J. M., Sauvaud, J. A., Cros, A., Dandouras, J., Aoustin, C., Bouyssou, J., Camus, T., Cuvilo, J., Martz, C., Médale, J. L., Perrier, H., Romefort, D., Rouzaud, J., D’Uston, C., Möbius, E., Crocker, K., Granoff, M., Kistler, L. M., Popecki, M., Hovestadt, D., Klecker, B., Paschmann, G., Scholer, M., Carlson, C. W., Curtis, D. W., Lin, R. P., Mcfadden, J. P., Formisano, V., Amata, E., Bavassano-Cattaneo, M. B., Baldetti, P., Belluci, G., Bruno, R., Chionchio, G., Di Lellis, A., Shelley, E. G., Ghielmetti, A. G., Lennartsson, W., Korth, A., Rosenbauer, H., Lundin, R., Olsen, S., Parks, G. K., Mccarthy, M., and Balsiger, H.: The Cluster ion spectrometry (CIS) experiment, Space Sci. Rev., 79, 303-350, 1997.

Sagdeev, R. Z.: Cooperative Phenomena and Shock Waves in Collisionless Plasmas, Rev. Plasma Phys., 4, 23-90, 1966.

Sagdeev, R. Z.: The 1976 Oppenheimer lectures: Critical problems in plasma astrophysics. II. Singular layers and reconnection, Rev. Modern Physics, 51, 11-20, doi:10.1103/RevModPhys.51.11, 1979.

Sagdeev, R. Z. and Galeev, A. A.: Nonlinear Plasma Theory, W.A. Benjamin, 1969.

Scholer, M., Shinohara, I., and Matsukiyo, S.: Quasi-perpendicular shocks: Length scale of the cross-shock potential, shock reformation, and implication for shock surfing, J. Geophys. Res., 108, 1014, doi:10.1029/2002JA009515, 2003.

Schwartz, S. J.: Shock and Discontinuity Normals, Mach Numbers, and Related Parameters, ISSI Scientific Reports Series, 1, 249270, 1998.

Scudder, J. D., Aggson, T. L., Mangeney, A., Lacombe, C., and Harvey, C. C.: The resolved layer of a collisionless, high beta, supercritical, quasi-perpendicular shock wave. I - RankineHugoniot geometry, currents, and stationarity, J. Geophys. Res., 91, 11019-11052, doi:10.1029/JA091iA10p11019, 1986.

Walker, S. N., Alleyne, H. St. C. K., Balikhin, M. A., André, M., and Horbury, T. S.: Electric field scales at quasi-perpendicular shocks, Ann. Geophys., 22, 2291-2300, doi:10.5194/angeo-222291-2004, 2004.

Walker, S. N., Balikhin, M. A., Alleyne, H. St. C. K., Hobara, Y., André, M., and Dunlop, M. W.: Lower hybrid waves at the shock front: a reassessment, Ann. Geophys., 26, 699-707, doi:10.5194/angeo-26-699-2008, 2008.

Woolliscroft, L. J. C., Alleyne, H. S. C., Dunford, C. M., Sumner, A., Thompson, J. A., Walker, S. N., Yearby, K. H., Buckley, A., Chapman, S., and Gough, M. P.: The Digital Wave-Processing Experiment on Cluster, Space Sci. Rev., 79, 209-231, 1997.

Wu, C. S.: A Fast Fermi Process: Energetic Electrons Accelerated by a Nearly Perpendicular Bow Shock, J. Geophys. Res., 89, 8857-8862, doi:10.1029/JA089iA10p08857, 1984.

Wygant, J. R., Bensadoun, M., and Mozer, F. S.: Electric field measurements at subcritical, oblique bow shock crossings, J. Geophys. Res., 92, 11109-11121, doi:10.1029/JA092iA10p11109, 1987. 